\title{
Decoherence effect in neutrinos produced in microquasar jets
}

\author{
M. E. Mosquera, ${ }^{a, b}$ O. Civitarese $e^{b, 1}$
}

${ }^{a}$ Facultad de Ciencias Astronómicas y Geofísicas, Universidad Nacional de La Plata, Paseo del Bosque, (1900) La Plata, Argentina

${ }^{b}$ Department of Physics, University of La Plata, c.c. 67 (1900), La Plata, Argentina

E-mail: mmosquera@fcaglp.unlp.edu.ar,osvaldo.civitarese@fisica.unlp.edu.ar

Abstract. We study the effect of decoherence upon the neutrino spectra produced in microquasar jets. In order to analyse the precession of the polarization vector of neutrinos we have calculated its time evolution by solving the corresponding equations of motion, and by assuming two different scenarios, namely: (i) the mixing between two active neutrinos, and (ii) the mixing between one active and one sterile neutrino. We have found that for the case with two active neutrinos and large values of the neutrino-neutrino interactions the onset of decoherence is not manifest. For the active-sterile scheme decoherence becomes manifest if the strength of the neutrino-neutrino interactions $(\mu)$ and the ratio between the square-mass difference and the energy $\left(\omega=\frac{\delta m^{2}}{2 E}\right)$ satisfy the relation $\frac{\mu}{w_{E=E_{\min }}}>0.1$.

Keywords: decoherence, neutrino fluxes, microquasars

\footnotetext{
${ }^{1}$ Corresponding author.
} 


\section{Contents}

1 Introduction $\quad 1$

2 Formalism 2

3 Neutrino spectra 3

3.1 Gaussian spectrum 3

3.2 Jet's neutrino spectrum in microquasar from $p \gamma$ and $p p$ interactions 4

3.2.1 Proton injection 5

3.2.2 Pion injection 5

3.2.3 Muon injection 5

3.2.4 Neutrino injection 5

3.3 Jet's neutrino spectrum in windy microquasar 6

4 Results $\quad 8$

4.1 Gaussian spectra 8

$\begin{array}{llr}4.2 & \text { Two-active neutrinos } & 9\end{array}$

$\begin{array}{lll}4.3 & \text { Active-sterile neutrino } & 10\end{array}$

5 Conclusions 11

\section{Introduction}

The study of neutrino's related processes in astroparticle physics is a subject of utmost importance since it is strongly connected with crucial aspects of particle physics [1]. The achievements in the field, both theoretically and experimentally are impressive: the values of the neutrino-flavor oscillations parameters have been determined [2-5] and various scenarios for the mass hierarchy have been proposed and constrained experimentally [6]. Lately, the importance of neutrino-neutrino interactions in star evolution has been emphasized [7, 8], particularly in dealing with supernovae's dynamics. The neutrinos are indeed very peculiar particles, since they can travel enormous distances without being severely affected by local interactions. However, their quantum nature should manifests in phenomena like decoherence [9-11]. As pointed out in Ref. [12], the onset of decoherence may affect strongly the density and energy-momentum distribution of neutrinos produced in distant sources.

As it is well known from elementary quantum mechanics, pure states may evolve into mixed states due to interactions with the background $[9,10]$. If this is the case with neutrinos produced in supernovae explosions or in other astrophysical events the information about oscillation parameters, masses, etc, may be depending upon the presence of decoherence. In this paper we focus on neutrinos produced from various reactions which take place in a microquasar. By modelling their spectra and initial densities we are able to follow their evolution in time and determine conditions for the appearance of decoherence. We have followed the formalism of $[13,14]$ and adapted it to calculate the pattern of decoherence in the time evolution of microquasar's neutrinos. We have found a dependence of the decoherence pattern with the mixing scheme of the neutrinos and with the neutrino-neutrino interactions. 
We have considered two cases, that is a) the mixing between active neutrinos and b) the mixing between active and sterile neutrinos.

The work is organized as follows. In Section 2 we present the formalism which we have developed to calculate the time evolution of the neutrino spectra and in Section 3 we used it to obtain the neutrino spectrum in a microquasar jet. The results of the calculations are presented in Section 4. Our conclusions are drawn in Section 5.

\section{Formalism}

The time evolution of the occupation number of neutrinos is governed by the equation of motion [12]

$$
\imath \dot{\rho_{f}}=\left[\frac{M^{2} c^{4}}{2 E \hbar}+\sqrt{2} G_{F} \rho, \rho_{f}\right]
$$

where the squared brackets reads for the commutator, the dot represents the time derivate and $M^{2} c^{4}$ is the mass-squared matrix in the flavour basis. The quantity $\rho_{f}$ is the density matrix in the flavour basis, and $\rho$ is a matrix whose diagonal terms are the neutrino number densities, $E$ stands for the neutrino energy and $G_{F}$ is the Fermi constant.

The mass-squared matrix in the flavour basis can be obtained by a transformation of the mass-squared matrix in the mass basis $\left(m^{2} c^{4}\right)$ through the unitary mixing matrix $U$. That is:

$$
\begin{aligned}
M^{2} c^{4}= & U m^{2} c^{4} U^{\dagger} \\
= & \frac{1}{2}\left(m_{1}^{2}+m_{2}^{2}\right) c^{4}\left(\begin{array}{ll}
1 & 0 \\
0 & 1
\end{array}\right)+\frac{1}{2} \sin 2 \theta\left(m_{2}^{2}-m_{1}^{2}\right) c^{4}\left(\begin{array}{ll}
0 & 1 \\
1 & 0
\end{array}\right) \\
& -\frac{1}{2} \cos 2 \theta\left(m_{2}^{2}-m_{1}^{2}\right) c^{4}\left(\begin{array}{cc}
1 & 0 \\
0 & -1
\end{array}\right) .
\end{aligned}
$$

In the above equation $m_{1}$ and $m_{2}$ stand for the mass of the neutrino in the mass eigenstate $\nu_{1}$ and $\nu_{2}$, respectively.

Following Ref. [12], one can write the mass matrix and the matrix $\rho_{f}$ in terms of Pauli matrices. For two-neutrino mass eigenstates they are written

$$
\begin{aligned}
\frac{M^{2} c^{4}}{2 E \hbar} & =\frac{1}{2} \operatorname{tr}\left(\frac{m^{2} c^{4}}{2 E \hbar}\right) I+\frac{1}{2} w \bar{B} \cdot \bar{\sigma}, \\
\rho_{f} & =\frac{1}{2} \operatorname{tr}\left(\rho_{f}\right) I+\frac{1}{2} \bar{P}_{f} \cdot \bar{\sigma} .
\end{aligned}
$$

where $\bar{P}_{f}$ is the polarization vector in the flavour basis, $w=\frac{\delta m^{2}}{2 E \hbar}$, and $\delta m^{2}=m_{2}^{2} c^{4}-m_{1}^{2} c^{4}$ is the mass-squared difference between the mass eigenstates, $\bar{B}$ is an unitary vector which fixes the orientation of the background. This vector, by simple comparison between the Eqs. (2.2) and (2.3), is a column vector with components

$$
\bar{B}=\left(\begin{array}{c}
\sin 2 \theta \\
0 \\
-\cos 2 \theta
\end{array}\right) .
$$


One can performed a rotation of the coordinate system in the flavour space and choose the direction of $\bar{B}$ in the $\mathrm{z}$ axis. This direction does not necessary correspond to the external physical magnetic field. The equation of motion is then re-written as

$$
\frac{\partial \bar{P}_{w}}{\partial t}=(w \bar{B}+\mu \bar{P}) \times \bar{P}_{w}
$$

where $\mu$ stands for the neutrino-neutrino interaction ${ }^{1}$ and

$$
\bar{P}=\int \bar{P}_{w} d w
$$

is the total (or global) polarizarion vector. Then the initial condition for $\bar{P}_{w}$, is given by

$$
\bar{P}_{w}(0)=\left(\begin{array}{c}
\sin 2 \theta \\
0 \\
\cos 2 \theta
\end{array}\right) g(w)
$$

where $g(w)=A\left(g_{\mu}-g_{e}\right), g_{e}$ and $g_{\mu}$ are the electron and muon-neutrino spectral functions, $A$ is a normalization constant and $\theta$ is the neutrino mixing angle.

The order parameter that measures coherence is defined as the ratio between the modulus of the perpendicular polarization vector at time $t$ and it at time $t=0$, that is:

$$
R_{\theta}(t)=\frac{\left|\bar{P}_{\perp}(t)\right|}{\left|\bar{P}_{\perp}(0)\right|}
$$

where $\bar{P}_{\perp}(t)=\bar{P}-(\bar{P} \cdot \bar{B}) \bar{B}$. The average in angles can be computed as

$$
R(t)=\frac{\int R_{\theta}(t) \mathrm{d} \theta}{\int \mathrm{d} \theta}
$$

\section{Neutrino spectra}

\subsection{Gaussian spectrum}

Following Ref. [12] and as toy models we shall use two different Gaussian-like spectra

$$
\begin{aligned}
& g_{w 1}(w)=\frac{1}{2 \sqrt{2 \pi}} e^{-(w-5)^{2} / 2}+\frac{1}{2 \sqrt{2 \pi}} e^{-(w+5)^{2} / 2}, \\
& g_{w 2}(w)=\frac{1}{2 \sqrt{2 \pi}} e^{-(w-1)^{2} / 2}+\frac{1}{2 \sqrt{2 \pi}} e^{-(w+1)^{2} / 2},
\end{aligned}
$$

that is two-non-overlapping Gaussian distributions, $g_{w 1}$, and two-overlapping Gaussian functions, $g_{w 2}$, respectively.

\footnotetext{
${ }^{1}$ In the calculations we shall take different values of the coupling constant $\mu$, noticing that in [12] the calculations have been performed for some fixed values of it.
} 


\subsection{Jet's neutrino spectrum in microquasar from $p \gamma$ and $p p$ interactions}

In order to compute the neutrino spectrum produced in a microquasar, we follow Ref. [15]. We have assumed a compact object with an accretion disk and a perpendicular jet with a half-opening angle $\xi$. The injection point is located at $z_{0}$. In Table 1 we show the parameters used in the calculation. The differential equation that gives the density of the particles, $N(E)$, in a microquasar jet is

$$
\frac{\partial(N(E) b(E))}{\partial E}+\left(t_{d e c}^{-1}+t_{e s c}^{-1}\right) N(E)=Q(E)
$$

where $b(E)=-E t_{\text {loss }}^{-1}, Q(E)$ is the particle injection, $t_{\text {dec }}^{-1}$ and $t_{\text {esc }}^{-1}$ are the decay and escape rate respectively. The scape rate can be computed as $t_{\text {esc }}^{-1}=c /\left(z_{\max }-z\right)$, where $c$ is the speed of light, $z_{\max }$ is the extent of the acceleration region (that is $5 \times 10^{8} \mathrm{~cm}$ ), and $z$ is the position. The rate $E t_{\text {loss }}^{-1}$ is the sum of the cooling rates, that is $t_{s y n}^{-1}+t_{a d}^{-1}+t_{p p}^{-1}+t_{p \gamma}^{-1}$ for protons, $t_{s y n}^{-1}+t_{a d}^{-1}+t_{\pi p}^{-1}+t_{\pi \gamma}^{-1}$ for pions and $t_{s y n}^{-1}+t_{a d}^{-1}+t_{i c}^{-1}$ for muons, respectively. The other quantities needed to evalute the cooling rates are: $t_{s y n}^{-1}$ which is rate of emission of synchrotron radiation, $t_{a d}^{-1}$ which stands for the adiabatic cooling, $t_{p p}^{-1}$ which is the $p p$ collision rate, $t_{p \gamma}^{-1}$ which gives the rate of the interaction between protons and synchrotron photons. Finally, $t_{\pi p}^{-1}$ and $t_{\pi \gamma}^{-1}$ represent the proton-pion and pion-photon interaction rates and $t_{i c}^{-1}$ is the loss-rate of the inverse Compton interactions [15]. The solution of equation (3.2) is

$$
N(z, E)=\frac{1}{|b(z, E)|} \int_{E}^{E_{p}^{\max }} Q(z, x) e^{-\tau(E, x, z)} \mathrm{d} x
$$

where $E>1.2 \mathrm{GeV}$. The proton maximum energy $E_{p}^{\max }=5 \times 10^{6} \mathrm{GeV}$ is obtained through the assumption that the acceleration rate is equal to the loss rate at the initial high of the jet and

$$
\tau(E, x, z)=\int_{E}^{x} \mathrm{~d} y \frac{t_{d e c}^{-1}(y)+t_{e s}^{-1}(z)}{|b(z, y)|}
$$

Table 1. Microquasar parameters (some of the values where extracted from Ref. [15]).

\begin{tabular}{|c|c|}
\hline Parameter & Value \\
\hline Jet power & $5 \times 10^{40} \mathrm{erg} \mathrm{s}^{-1}$ \\
\hline Initial jet's high & $10^{8} \mathrm{~cm}$ \\
\hline Lorentz factor & 1.25 \\
\hline Relativistic particles & 0.1 \\
\hline Hadron-to-lepton ratio & 100 \\
\hline Half opening angle & 0.087 \\
\hline
\end{tabular}




\subsubsection{Proton injection}

The proton injection, $Q(z, E)$ of Eq.(3.3) is [15]

$$
Q(z, E)=Q_{0}^{p}\left(\frac{z_{0}}{z}\right)^{3} \Gamma^{-1}\left(E-\beta \sqrt{E^{2}-m_{p}^{2} c^{4}} \cos \theta\right)^{-2}\left(\Gamma-\cos \theta \frac{E \beta}{\sqrt{E^{2}-m_{p}^{2} c^{4}}}\right)
$$

$Q_{0}^{p}$ is a constant to be determined from the luminosity, $\Gamma$ is the Lorentz factor, $\theta$ is the observation angle and $\beta$ is related to $\Gamma$ (see [15] for details). In this case $t_{\text {dec }}^{-1}=0$.

\subsubsection{Pion injection}

The pion injection is $Q_{\pi}(E)=Q_{\pi}^{p p}(E)+Q_{\pi}^{p \gamma}(E)$, where $Q_{\pi}^{p p}(E)$ and $Q_{\pi}^{p \gamma}(E)$ are the injection terms resulting from the pion production due to proton-proton and proton-photon interactions, respectively. The proton-proton injection is calculated as

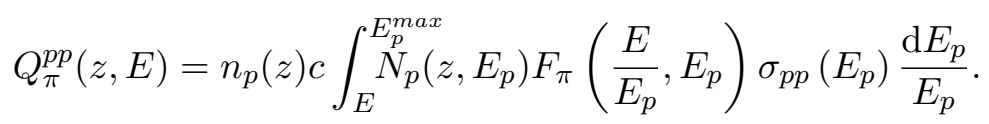

In the previous equation, $n_{p}$ is the density of cold particles, $N_{p}\left(z, E_{p}\right)$ is the proton density, $\sigma_{p p}$ is the cross section and $F_{\pi}$ is the pion's distribution produced per $p p$ collisions (see Ref. $[15,16])$

The proton-photon production is given by the expression

$$
Q_{\pi}^{p \gamma}(z, E)=5 N_{p}(z, 5 E, \theta) \omega_{p \gamma}(z, 5 E) \mathcal{N}_{\pi}(z, 5 E),
$$

where $\omega_{p \gamma}$ is the collision-frequency and $\mathcal{N}_{\pi}$ the mean number of positive and negative pions.

\subsubsection{Muon injection}

The pion decay produces muons, therefore the muon injection is

$$
\begin{aligned}
& Q_{\mu}^{L^{-} ; R^{+}}(z, E)=\int_{E}^{E_{p}^{\max }} t_{\operatorname{dec} \pi}^{-1}\left(E_{\pi}\right) N_{\pi}\left(z, E_{\pi}\right) \frac{\mathrm{d} n_{\pi^{-} \rightarrow \mu_{L}^{-}}}{\mathrm{d} E}\left(E, E_{\pi}\right) \mathrm{d} E_{\pi}, \\
& Q_{\mu}^{R^{-} ; L^{+}}(z, E)=\int_{E}^{E_{p}^{\max }} t_{\operatorname{dec} \pi}^{-1}\left(E_{\pi}\right) N_{\pi}\left(z, E_{\pi}\right) \frac{\mathrm{d} n_{\pi^{-} \rightarrow \mu_{R}^{+}}}{\mathrm{d} E}\left(E, E_{\pi}\right) \mathrm{d} E_{\pi} .
\end{aligned}
$$

In the previous expression $t_{d e c \pi}^{-1}$ is the pion decay rate, $N_{\pi}\left(z, E_{\pi}\right)$ the density of pions and $\frac{\mathrm{d} n_{\pi^{-} \rightarrow \mu_{L}^{-}}}{\mathrm{d} E}$ and $\frac{\mathrm{d} n_{\pi^{-} \rightarrow \mu_{R}^{+}}}{\mathrm{d} E}$ are the decay rates of left-handed and right-handed muons, respectively [17].

\subsubsection{Neutrino injection}

The neutrino production due to pion decay can be written as

$$
Q_{\pi \rightarrow \nu}(z, E)=\int_{E}^{E_{p}^{\max }} t_{\operatorname{dec} \pi}^{-1}\left(E_{\pi}\right) N_{\pi}\left(z, E_{\pi}\right) \Theta\left(1-\frac{E}{E_{\pi}}-\left(\frac{m_{\mu}}{m_{\pi}}\right)^{2}\right)\left(1-\left(\frac{m_{\mu}}{m_{\pi}}\right)^{2}\right)^{-1} \frac{\mathrm{d} E_{\pi}}{E_{\pi}},
$$


The neutrino injection due to the muon decay is

$$
Q_{\mu \rightarrow \nu}(z, E)=\sum_{i=1}^{4} \int_{E}^{E_{p}^{\max }} t_{d e c \mu}^{-1}\left(E_{\mu}\right) N_{\mu_{i}}\left(z, E_{\mu},\right) y\left(\frac{E}{E_{\mu}}\right) \frac{\mathrm{d} E_{\mu}}{E_{\mu}},
$$

where $t_{d e c \mu}^{-1}$ is the muon decay rate, $N_{\mu_{i}}\left(z, E_{\mu},\right)$ the muon density and $y(x)$ is a polynomial function [15].

The neutrino spectral function can be calculated by performing the integral in the jet volume

$$
\begin{aligned}
g_{\mu}\left(E_{\nu}\right) & =\int \mathrm{d} V \frac{Q_{\mu \rightarrow \nu}(z, E)+Q_{\pi \rightarrow \nu}(z, E)}{t_{e s c}^{-1}(z)} \\
g_{e}\left(E_{\nu}\right) & =\int \mathrm{d} V \frac{Q_{\mu \rightarrow \nu}(z, E)}{t_{e s c}^{-1}(z)}
\end{aligned}
$$

\subsection{Jet's neutrino spectrum in windy microquasar}

In order to calculate the neutrino density as a function of the energy, we have assumed that a binary system formed by one high-mass primary star sub-rounded by a disk and a compact object describing a Kepler-orbit (see Table 2) and followed the analysis presented in Ref.[13]. The jet of relativistic particles produced by the compact object is considered to be cone perpendicular to the accretion-disk plane (or orbital plane). We have used the wind velocity model [14]

$$
v\left(r_{w}\right)=v_{\infty}\left(\frac{R_{\star}}{r_{w}}\right)^{1.2}
$$

where $r_{w}$ is the radial coordinate from the center of the star, $R_{\star}$ is the star radius, $v_{\infty}$ is the terminal velocity of the wind. The mass density of the wind is obtained from the continuity equation [12].

Following Ref. [14] one can write the proton spectrum in the jet frame and the accretion rate due to the wind to obtain the proton flux in the observer frame $[13,14]$. These protons interact with target protons of the wind via the reaction

$$
p+p \rightarrow p+p+\xi_{\pi^{0}}\left(E_{P}\right) \pi^{0}+\xi_{\pi}\left(E_{P}\right)\left(\pi^{+}+\pi^{-}\right),
$$

where $\xi_{\pi^{0}}\left(E_{P}\right)$ and $\xi_{\pi}\left(E_{P}\right)$ are the multiplicities for neutral and charged pions, given by [13]

$$
\begin{aligned}
\xi_{\pi^{0}}\left(E_{p}\right) & =1.1\left(\frac{E_{p}}{\mathrm{GeV}}\right)^{1 / 4}, \\
\xi_{\pi}\left(E_{p}\right) & =\left(\frac{E_{p}}{\mathrm{GeV}}-1.22\right)^{1 / 5} .
\end{aligned}
$$

The proton $\left(E_{p}\right)$ and photon $\left(E_{\gamma}\right)$ energies are related by $E_{p}=6 k^{-1} \xi_{\pi^{0}}\left(E_{p}\right) E_{\gamma}$, where $k=0.5$ is the inelasticity coefficient.

From the energy conservation one can obtain the neutrino intensity produced by pionand muon-decay $[18,19]$

$$
\int_{E_{\gamma}^{\min }}^{E_{\gamma}^{\max }} \mathrm{d} E_{\gamma} \frac{\mathrm{d} N_{\gamma}}{\mathrm{d} E_{\gamma}} E_{\gamma}=\Delta \int_{E_{\nu}^{\min }}^{E_{\nu}^{\max }} \mathrm{d} E_{\nu} \frac{\mathrm{d} N_{\nu}}{\mathrm{d} E_{\nu}} E_{\nu}
$$


Table 2. Windy microquasar parameters (from Ref.[13]).

\begin{tabular}{|c|c|}
\hline Parameter & Value \\
\hline$M_{\star}$ & $10 R_{\odot}$ \\
\hline$M_{\text {compact object }}$ & $1.4 R_{\odot}$ \\
\hline$R_{\star}$ & $10 R_{\odot}$ \\
\hline Period & $22.8925 \times 10^{4} \mathrm{~s}$ \\
\hline Eccentricity & 0.72 \\
\hline Initial orbital phase & 0.261799 \\
\hline$\rho_{0}$ & $10^{-11} \mathrm{gr} \mathrm{cm}^{-3}$ \\
\hline$v_{\infty}$ & $5 \times 10^{5} \mathrm{~cm} \mathrm{~s}^{-1}$ \\
\hline Initial jet's high & $10^{7} \mathrm{~cm}^{\prime}$ \\
\hline Initial jet's radius & $10^{6} \mathrm{~cm}$ \\
\hline Proton spectrum power law & 2.2 \\
\hline Lorentz factor & 1.25 \\
\hline
\end{tabular}

where $E_{\gamma}^{\min }$ and $E_{\gamma}^{\max }$ are the minimum and maximum energies of photons resulting from hadrons, and $E_{\nu}^{\min }$ and $E_{\nu}^{\max }$ are the corresponding minimum and maximum energy of the neutrinos, and $\Delta=1$ [19]. The neutrino energy is related to the photon energy by $E_{\nu}=\frac{1}{2} E_{\gamma}$, leading to [20]

$$
E_{\nu}=\frac{k}{12 \xi_{\pi}\left(E_{p}\right)} E_{p}
$$

The maximum neutrino energy is determined by the maximum energy acquired by the accelerated protons, which is related to the magnetic field $B$. The magnetic field is calculated by assuming equipartition between the magnetic field energy and the kinetic energy of the jet [13]. The maximum energy of the protons is

$$
E_{p}(\psi)=e R\left(z_{0}\right) B\left(\psi, z_{0}\right) .
$$

Note that $0.5 \mathrm{GeV}<E_{p}(\psi)<2.8 \times 10^{4} \mathrm{GeV}$.

The muon-neutrino density computed as

$$
G_{\mu}\left(E_{\nu}, \psi\right)=\frac{4 f_{p}}{m_{p}} \int \mathrm{d} V \frac{\rho_{w}(\psi ; z, \delta, \phi) q_{\gamma}\left(\psi ; 2 E_{\nu}, z, \theta\right)}{t_{e s c}^{-1}(z)},
$$

where $f_{p}=0.1$ takes into account particle-rejection from the boundary [21], $\rho_{w}(\psi ; z, \delta, \phi)$ is the wind mass density, $q_{\gamma}\left(\psi ; 2 E_{\nu}, z, \theta\right)$ stands for the gamma-ray emissivity [22] and $t_{e s c}^{-1}(z)=$ $\frac{c}{z_{m}-z}$ is the inverse of the neutrino's escape time. The integral is performed in the jet's volume.

The muon neutrino spectrum is then calculated as

$$
g_{\mu}(w)=\frac{1}{2 \pi} \int_{0}^{2 \pi} \mathrm{d} \psi G_{\mu}\left(E_{\nu}, \psi\right) .
$$

The electron-neutrino spectrum can be determined by repeating the same arguments. 


\section{Results}

We have considered two different neutrino's scenario, that is: (i) two-active neutrinos and one active and (ii) one sterile neutrino, to compute the order parameter. The neutrino-mixing parameters for the first scenario are $\delta m^{2}=7.53 \times 10^{-5} \mathrm{eV}^{2}$ and $\sin ^{2} \theta=0.307$ [23]. In this case, we have computed both the electron- and muon-neutrino spectral functions produced in the microquasar and used them as initial condition (see Eq. 2.8) to compute the time dependence of the polarization vector and the order parameter. For the active-sterile neutrino mixing parameters we have used $\delta m^{2}=1 \mathrm{eV}^{2}$ and $\sin ^{2} \theta=0.1$. As initial condition for the sterile neutrino sector we assume that sterile neutrino are not produced in the microquasar jet.

\subsection{Gaussian spectra}

As toy model we have computed the order parameter for two-active neutrino's Gaussian spectrum. In Figure 1 we show the order parameter for the two-Gaussian spectra at the mixing angle (top figure) and its mean value (bottom figure), as a function of the time and for different values of $\mu$. In absence of neutrino-neutrino interactions, that is $\mu=0$, the order parameter shrinks to zero as well as its mean value. When the neutrino-neutrino interaction is activated $|\bar{P}|$ decreases its value but oscillates around a non-zero value. The larger the value of the interaction the larger is $|\bar{P}|$. The mean value of the order parameter reaches a smaller average value earlier than the one calculated with a fixed mixing angle.
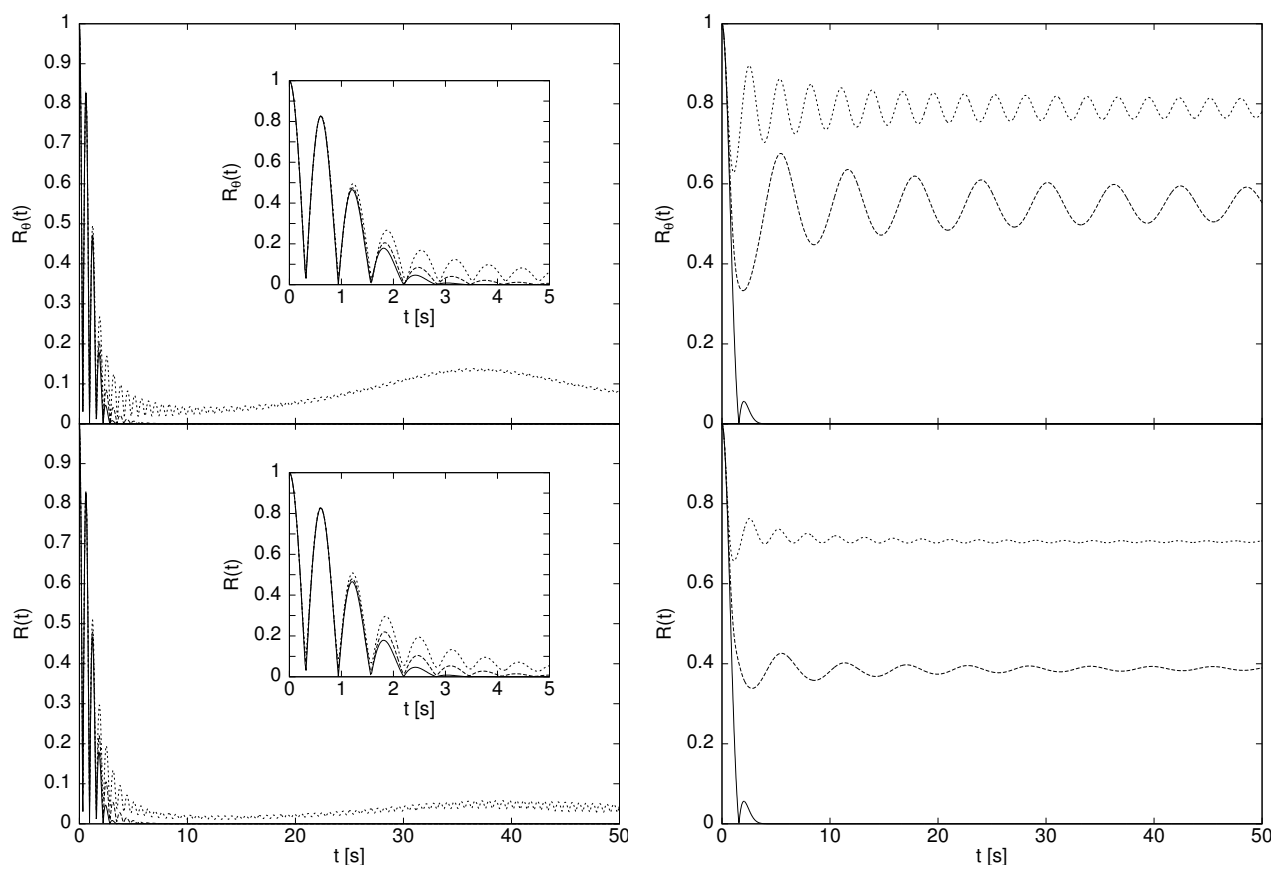

Figure 1. Left figure: order parameter as a function of the time, for two-separated Gaussian spectra. Top figure: order parameter using the active-active mixing angle; bottom figure: mean value of the order parameter. Solid line: $\mu=0 \mathrm{~s}^{-1}$; dashed line: $\mu=1 \mathrm{~s}^{-1}$; dotted line: $\mu=1.8 \mathrm{~s}^{-1}$. Right figure: order parameter as a function of the time, for two-overlapping Gaussian spectra. Top figure: order parameter using the active-active mixing angle; bottom figure: mean value of the order parameter. Solid line: $\mu=0 \mathrm{~s}^{-1}$; dashed line: $\mu=2 \mathrm{~s}^{-1}$; dotted line: $\mu=3 \mathrm{~s}^{-1}$. 
The results for the case of two-overlapping Gaussian as initial condition of the neutrino spectrum, shown at the right inset of Figure 1, are similar to the ones obtained in Ref. [12] for a Gaussian spectrum.

\subsection{Two-active neutrinos}

In this section we present the results for the order parameter calculated by using the neutrino spectral function as described in Section 3.2 and in Section 3.3. The results for the first case are shown in the left inset of Figure 2. They are similar to the ones obtained using as initial condition the two-non-overlapping Gaussian functions. The length of the vector $\bar{P}$ is reduced to zero for the non-interacting case and for small values of $\mu$. However, for larger values of the neutrino-neutrino interaction, the polarization vector oscillates towards an asymptotic non-zero value.
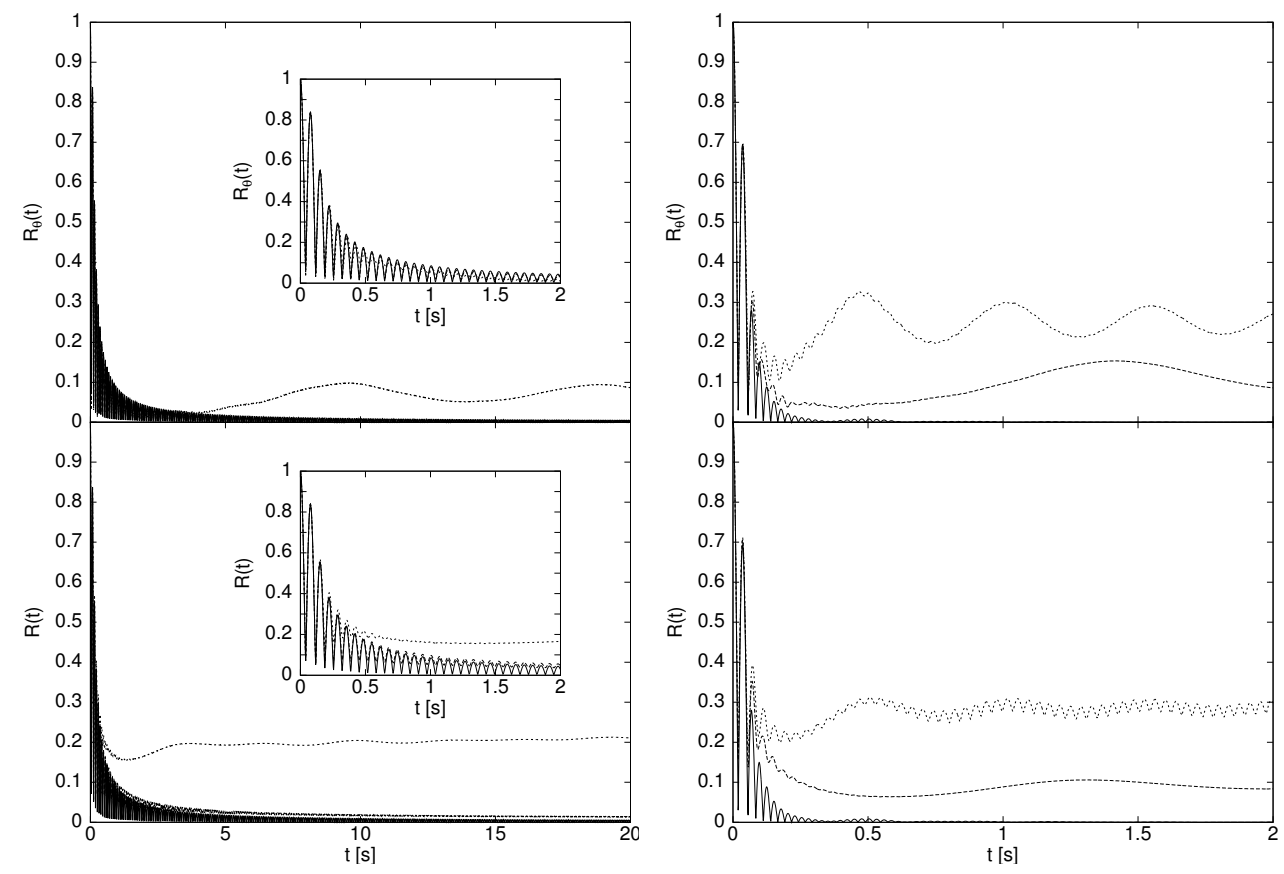

Figure 2. Left figure: order parameter as a function of time calculated from the emission of neutrinos in a microquasar's jet and two-active neutrino. Top figure: order parameter using the active-active mixing angle; bottom figure: mean value of the order parameter. Solid line: $\mu=0 \mathrm{~s}^{-1}$; dashed line: $\mu / w_{\max }=0.08$; dotted line $\mu / w_{\max }=0.2$. Right figure: order parameter as a function of time calculated from the neutrino spectrum in a windy microquasar and two-active neutrino. Top figure: order parameter using the active-active mixing angle; bottom figure: mean value of the order parameter. Solid line: $\mu=0 \mathrm{~s}^{-1}$; dashed line: $\mu / w_{\max }=0.32$; dotted line $\mu / w_{\max }=0.40$.

The order parameter $R$ obtained using the neutrino spectra described in section 3.3 is shown in the right inset of Figure 2, for active-active neutrino scheme.

The time that neutrinos remain inside the jet can be computed as $R_{\max } / c$. For the calculation using the formalism describe in Section 3.2, this time is $1.4 \times 10^{-3} \mathrm{~s}$ and in windy microquasar is $1.7 \times 10^{-4} \mathrm{~s}$. Therefore, inside the microquasar's jet, the decoherence has not effect in the neutrino distribution. 


\subsection{Active-sterile neutrino}

For the active-sterile neutrino, the results are shown in Figure 3. The results displayed in the left inset of this figure have been obtained by applying the formalism described in section 3.2. As seen from the curves, the length of the vector $\bar{P}$ is reduced to zero for the non-interacting case but it does not vanishes for larger values of the neutrino-neutrino interaction.
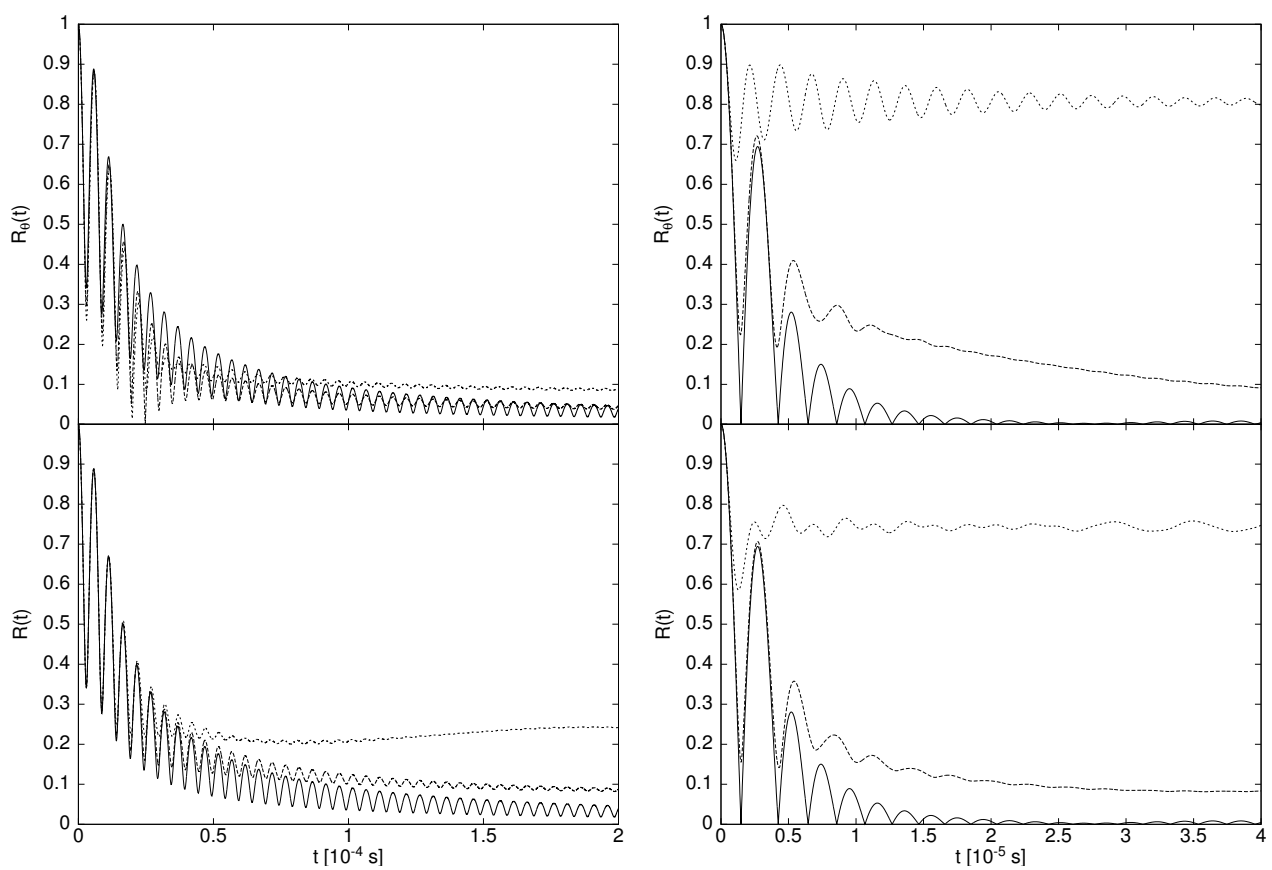

Figure 3. Left figure: order parameter as a function of the time calculated from the emission of neutrinos in a microquasar's jet and for active-sterile neutrino scheme. Top figure: order parameter using the active-active mixing angle; bottom figure: mean value of the order parameter. Solid line: $\mu=0 \mathrm{~s}^{-1}$; dashed line: $\mu / w_{\max }=0.1$; dotted line $\mu / w_{\max }=0.16$. Right figure: order parameter as a function of the time calculated from the neutrino spectrum in a windy microquasar and active-sterile neutrino scheme. Top figure: order parameter using the active-active mixing angle; bottom figure: mean value of the order parameter. Solid line: $\mu=0 \mathrm{~s}^{-1}$; dashed line: $\mu / w_{\max }=0.33$; dotted line $\mu / w_{\max }=1.25$.

For the windy microquasar jet formalism one can see that, for large values of the parameter $\mu$, the length of the polarization vector is reduced by a $20 \%$, as shown in the right inset of Figure 3. Meanwhile, for small values of the interaction, the length of the vector $\bar{P}$ is depleted. In this case, inside the microquasar's jet, the neutrino distribution are strongly affected by the decoherence, since the time of stay of neutrino in the jet is $O\left(10^{-3}\right) \mathrm{s}$, for microquasar's jet and $O\left(10^{-4}\right) \mathrm{s}$, for windy microquasar formalism.

Some final comments about the onset of decoherence, for the cases which we have considered in this section. For the case of only active neutrinos, if one fixes the neutrino boundaries in the region of the order of $10^{8} \mathrm{~cm}$ the time they spend there is of the order of $1.6 \times 10^{-5}$ seconds, a time shorter than the time needed to evolve into pointer states (see Figure 2). However, if the coupling with sterile neutrinos is activated the onset of decoherence becomes clear from the results (see Figure 3). The same behaviour is then expected for the outside region. A more precise determination of the effect requires the comparison between the length 
of the flavor oscillations and the length of decoherence.

\section{Conclusions}

In this work we have studied the effect of collective oscillations upon the neutrino spectral function, for neutrinos produced in micro quasar's jets, by applying the formalism developed in Refs. $[13,15]$. Using active neutrinos as initial condition for the evolution of the polarization vector $\bar{P}$ we have calculated the order parameter $R_{\theta}$ as a function of the time.

For the case of neutrino's Gaussian spectra we have found that the polarization vector reduces its length to zero for small or null neutrino interactions, exhibiting a complete decoherence-pattern. If the neutrino density is large enough the length of the polarization vector becomes smaller than one and it oscillates around a non-zero asymptotic value. For non-overlapping Gaussians the reduction of the order parameter is faster and the neutrino density must increase in order to reduce the effects of decoherence.

The results using the realistic electron- and muon-neutrino spectra produced in a micro quasar's jet are quite similar, for both formalisms [13, 15], and similar to the ones obtained with the toy (Gaussian) models. The polarization vector is reduced to zero for small neutrino density while for larger values of the neutrino-neutrino interactions, the decoherence is not completed. This effect is quite noticeable, e.g. for $\mu / w_{\max }=0.40$, which results in a mean value of the order parameter equals to 0.3 at large times. However, the decoherence inside the microquasar's jet do not have a strong effect upon the polarization vector.

When a sterile neutrino is oscillating with a light active-neutrino (electron-neutrino) the effects of the collective oscillations are noticeable at very small times, since the maximum value for the frequency $w$ is quite large due to the mass difference. In this case, for the electronneutrino spectral function calculated in Section 3.2, the decoherence is almost complete for the mixing angle used, but the mean value of the order parameter is different from zero for $\mu / w_{\max }>0.1$. For the case of a windy microquasar the polarization vector is reduced and this reduction is smaller if the neutrino density is larger. Inside the jet, in this scheme, the decoherence strongly affects the neutrino distribution function.

Finally, the effect of decoherence is present in the neutrino flux produced in microquasar jets, with or without sterile neutrino. This effect is noticeable for small values of the neutrino density. For active-sterile neutrino mixing the effect becomes noticeable at earlier times than for the mixing between active neutrinos.

\section{Acknowledgments}

This work was supported by a grant (PIP-282,-616) of the National Research Council of Argentina (CONICET), and by a research-grant of the National Agency for the Promotion of Science and Technology (ANPCYT) of Argentina. The authors are members of the Scientific Research Career of the CONICET.

\section{References}

[1] R. N. Mohapatra and A. Y. Smirnov, Neutrino Mass and New Physics, Annual Review of Nuclear and Particle Science 56 (2006) 569-628, [hep-ph/0603118].

[2] D. V. Forero, M. Tórtola, and J. W. F. Valle, Neutrino oscillations refitted, Phys. Rev. D 90 (2014) 093006, [arXiv: 1405.7540]. 
[3] M. C. Gonzalez-Garcia, M. Maltoni, and T. Schwetz, Updated fit to three neutrino mixing: status of leptonic CP violation, Journal of High Energy Physics 11 (2014) 52, [arXiv: 1409.5439].

[4] F. Capozzi, E. Lisi, and A. Marrone, Neutrino mass hierarchy and electron neutrino oscillation parameters with one hundred thousand reactor events, Phys. Rev. D 89 (2014) 013001, [arXiv: 1309.1638].

[5] F. Capozzi, E. Lisi, A. Marrone, D. Montanino, and A. Palazzo, Neutrino masses and mixings: Status of known and unknown 32 parameters, Nuclear Physics B 908 (2016) 218-234, [arXiv: 1601 .0777].

[6] X. Qian and P. Vogel, Neutrino mass hierarchy, Progress in Particle and Nuclear Physics $\mathbf{8 3}$ (2015) 1-30, [arXiv:1505.0189].

[7] I. Tamborra, G. Raffelt, F. Hanke, H.-T. Janka, and B. Müller, Neutrino emission characteristics and detection opportunities based on three-dimensional supernova simulations, Phys. Rev. D 90 (2014) 045032, [arXiv: 1406.0006].

[8] I. Tamborra, F. Hanke, H.-T. Janka, B. Müller, G. G. Raffelt, and A. Marek, Self-sustained Asymmetry of Lepton-number Emission: A New Phenomenon during the Supernova Shock-accretion Phase in Three Dimensions, ApJ 792 (2014) 96, [arXiv: 1402.5418].

[9] M. Schlosshauer, Decoherence, the measurement problem, and interpretations of quantum mechanics, Reviews of Modern Physics 76 (2004) 1267-1305, [quant-ph/0312059].

[10] W. H. Zurek, Pointer basis of quantum apparatus: Into what mixture does the wave packet collapse?, Phys. Rev. D 24 (1981) 1516-1525.

[11] W. H. Zurek, Environment-induced superselection rules, Phys. Rev. D 26 (1982) 1862-1880.

[12] G. G. Raffelt and I. Tamborra, Synchronization versus decoherence of neutrino oscillations at intermediate densities, Phys. Rev. D 82 (2010) 125004, [arXiv: 1006.0002].

[13] H. R. Christiansen, M. Orellana, and G. E. Romero, High-energy neutrino emission from x-ray binaries, Phys. Rev. D 73 (2006) 063012, [astro-ph/0509214].

[14] G. E. Romero, D. F. Torres, M. M. Kaufman Bernadó, and I. F. Mirabel, Hadronic gamma-ray emission from windy microquasars, A\&A 410 (2003) L1-L4, [astro-ph/0309123].

[15] M. M. Reynoso and G. E. Romero, Magnetic field effects on neutrino production in microquasars, A\&A 493 (2009) 1-11, [arXiv:0811.1383].

[16] S. R. Kelner, F. A. Aharonian, and V. V. Bugayov, Energy spectra of gamma rays, electrons, and neutrinos produced at proton-proton interactions in the very high energy regime, Phys. Rev. D 74 (2006) 034018, [astro-ph/0606058].

[17] P. Lipari, M. Lusignoli, and D. Meloni, Flavor composition and energy spectrum of astrophysical neutrinos, Phys. Rev. D 75 (2007) 123005, [arXiv:0704.0718].

[18] F. W. Stecker and M. H. Salamon, High Energy Neutrinos from Quasars, Space Sci. Rev. 75 (1996) 341-355, [astro-ph/9501064].

[19] J. Alvarez-Muñiz and F. Halzen, Possible High-Energy Neutrinos from the Cosmic Accelerator $R X J 1713.7-3946$, ApJ 576 (2002) L33-L36, [astro-ph/0205408].

[20] V. L. Ginzburg and S. I. Syrovatskii, The Secondary Electron Component of Cosmic Rays and the Spectrum of General Galactic Radio Emission, Soviet Ast. 8 (1964) 342.

[21] G. E. Romero, H. R. Christiansen, and M. Orellana, Hadronic High-Energy Gamma-Ray Emission from the Microquasar LS I +61 303, ApJ 632 (2005) 1093-1098, [astro-ph/0506735].

[22] F. A. Aharonian and A. M. Atoyan, On the emissivity of $\pi^{\wedge} 0^{\wedge}$-decay gamma radiation in the vicinity of accelerators of galactic cosmic rays., A\&A 309 (1996) 917-928. 
[23] C. Patrignani and Particle Data Group, Review of Particle Physics, Chinese Physics C 40 (2016) 100001. 